\title{
BONFERRONI-TYPE INEQUALITIES VIA INTERPOLATING POLYNOMIALS
}

\author{
YUAN XU
}

(Communicated by George C. Papanicolaou)

\begin{abstract}
Inequalitites of Bonferroni-type are proved by means of the corresponding polynomial inequalities which are obtained from the Hermite interpolation.
\end{abstract}

\section{INTRODUCTION}

Let $A_{1}, A_{2}, \ldots, A_{n}$ be events of a given probability space, and set $S_{0, n}=1$,

$$
S_{k, n}=\sum P\left(A_{i_{1}} \cap A_{i_{2}} \cap \cdots \cap A_{i_{k}}\right), \quad k \geqq 1,
$$

where the summation is over $1 \leqq i_{1}<i_{2}<\cdots<i_{k} \leqq n$. The numbers $S_{k, n}$ are the binomial moments of the number $m_{n}$ of occurrences amount the $A_{j}$. Linear inequalities of the form

$$
\sum c_{k} S_{k, n} \leqq P\left(m_{n}=0\right) \leqq \sum d_{k} S_{k, n}
$$

are equivalent to the polynomial inequalities

$$
\sum c_{k}\left(\begin{array}{l}
n \\
k
\end{array}\right) p^{k} \leqq(1-p)^{n} \leqq \sum d_{k}\left(\begin{array}{l}
n \\
k
\end{array}\right) p^{k}
$$

by a result of Galambos and Mucci [3]. The classical Bonferroni inequalities corresponding to $c_{k}=(-1)^{k}, 1 \leqq k \leqq 2 r-1$, and $c_{k}=0$ for $k \geqq 2 r$, and $d_{k}=(-1)^{k}, 1 \leqq k \leqq 2 r$, and $d_{k}=0$ for $k \geqq 2 r+1$. The corresponding polynomial inequality (2) is just a finite Taylor expansion of $(1-p)^{n}$, or from another point of view, this is the Hermite interpolating polynomial at one point $p=0$. This fact raises the question whether other interpolating polynomials provide better inequalities in (2) and thus in (1). This paper deals with such interpolating polynomial methods. In particular, we will show that the SobelUppuluri-Galambos inequalities

(3) $\sum_{k=1}^{2 t}(-1)^{k+1} S_{k, n}+\frac{2 t+1}{n} S_{2 t+1, n} \leqq P\left(m_{n}=0\right) \leqq \sum_{k=1}^{2 t-1}(-1)^{k+1} S_{k, n}-\frac{2 t}{n} S_{2 t, n}$

Received by the editors February 24, 1989.

1980 Mathematics Subject Classification (1985 Revision). Primary 62E99.

Key words and phrases. Bonferroni-type inequalities, Hermite interpolation. 
[2, Theorem 1.41] are given by a special two-point Hermite interpolating polynomial.

\section{HERMITE INTERPOLATING POLYNOMIALS}

For a given function $f$, we consider the two-point Hermite interpolating polynomial $H_{r, s}(f ; p)$ of degree $\leqq r+s-1$, which is uniquely defined by the following conditions:

$$
\begin{array}{ll}
H_{r, s}^{(q)}(f ; 0)=f^{(q)}(0), & 0 \leqq q \leqq r-1, \\
H_{r, s}^{(q)}(f ; 1)=f^{(q)}(1), & 0 \leqq q \leqq s-1 .
\end{array}
$$

The polynomial $H_{r, s}(f ; p)$ can conveniently be written in the form

$$
H_{r, s}(f ; p)=\sum_{k=0}^{r-1} T_{k}(p) f^{(k)}(0)+\sum_{k=0}^{s-1} R_{k}(p) f^{(k)}(1),
$$

where $T_{k}(p)$ is uniquely determined by

$$
T_{k}^{(j)}(0)=\delta_{j k}, \quad 0 \leqq j \leqq r-1 ; \quad T_{k}^{(j)}(1)=0, \quad 0 \leqq j \leqq s-1 .
$$

From (5), it can be easily verified that

$$
T_{k}(p)=(1-p)^{s} \sum_{k=0}^{r-k-1}\left(\begin{array}{c}
s+i-1 \\
i
\end{array}\right) p^{i+k} / k !
$$

Throughout this paper, we use notations $\left(\begin{array}{l}q \\ 0\end{array}\right)=1$ and $\left(\begin{array}{l}q \\ p\end{array}\right)=0, q<p$, or $p<0$. Now let $f_{n}(p)=(1-p)^{n}$; we can write $H_{r, s}\left(f_{n} ; p\right)$ into a more compact form.

Lemma 1. $H_{r, s+1}\left(f_{n} ; p\right)=(1-p) H_{r, s}\left(f_{n-1} ; p\right)$.

Proof. By (4) and (6)

$$
\begin{aligned}
(1-p)^{-s-1} H_{r, s+1}\left(f_{n} ; p\right)= & \sum_{k=0}^{r-1}(-1)^{k}\left(\begin{array}{c}
n \\
k
\end{array}\right) \sum_{i=0}^{r-k-1}\left(\begin{array}{c}
s+i \\
i
\end{array}\right) p^{i+k} \\
= & \sum_{k=0}^{r-1}(-1)^{k}\left(\begin{array}{c}
n-1 \\
k
\end{array}\right) \sum_{i=0}^{r-k-1}\left(\begin{array}{c}
s+i-1 \\
i
\end{array}\right) p^{k+i} \\
& +\sum_{k=0}^{r-1}(-1)^{k}\left(\begin{array}{c}
n-1 \\
k
\end{array}\right) \sum_{i=0}^{r-k-1}\left(\begin{array}{c}
s+i-1 \\
i-1
\end{array}\right) p^{k+i} \\
& +\sum_{k=0}^{r-1}(-1)^{k}\left(\begin{array}{c}
n-1 \\
k-1
\end{array}\right) \sum_{i=0}^{r-k-1}\left(\begin{array}{c}
s+1 \\
i
\end{array}\right) p^{i+k} \\
:= & I_{1}+I_{2}+I_{3},
\end{aligned}
$$


where $\left(\begin{array}{c}m \\ j\end{array}\right)=\left(\begin{array}{c}m-1 \\ j\end{array}\right)\left(\begin{array}{c}m-1 \\ j-1\end{array}\right)$ is used. Since it can be easily checked that $I_{2}=-I_{3}$, we get

$$
\begin{aligned}
(1-p)^{-s-1} H_{r, s+1}\left(f_{n} ; p\right) & =\sum_{k=0}^{r-1}(-1)^{k}\left(\begin{array}{c}
n-1 \\
k
\end{array}\right) \sum_{i=0}^{r-k-1}\left(\begin{array}{c}
s+i-1 \\
i
\end{array}\right) p^{k+i} \\
& =(1-p)^{-s} H_{r, s}\left(f_{n-1} ; p\right) .
\end{aligned}
$$

Using Lemma 1 repeatedly, we get

$$
H_{r, s}\left(f_{n} ; p\right)=(1-p)^{s} H_{r, 0}\left(f_{n-s} ; p\right),
$$

where by (4) and (5),

$$
H_{r, 0}\left(f_{n-s} ; p\right)=\sum_{k=0}^{r-1}(-1)^{k}\left(\begin{array}{c}
n-s \\
k
\end{array}\right) p^{k}
$$

Furthermore, we get from (7) and (8) that

$$
H_{r, s+1}\left(f_{n} ; p\right)-H_{r, s}\left(f_{n} ; p\right)=(1-p)^{s}(-1)^{r}\left(\begin{array}{c}
n-s-1 \\
r-1
\end{array}\right) p^{r}
$$

therefore,

$$
H_{2 m, s+1}\left(f_{n} ; p\right) \geqq H_{2 m, s}\left(f_{n} ; p\right), \quad H_{2 m+1, s+1}\left(f_{n} ; p\right) \leqq H_{2 m+1, s}\left(f_{n} ; p\right) .
$$

From the remainder formula of Hermite interpolation (cf. [1]), we get

$$
\begin{aligned}
f_{n}(p)-H_{r, s}\left(f_{n} ; p\right) & =\frac{p^{r}(p-1)^{s}}{(r+s) !} f_{n}^{(r+s)}(\zeta) \\
& =(-1)^{r}\left(\begin{array}{c}
n \\
r+s
\end{array}\right) p^{r}(1-p)^{s}(1-\zeta)^{n-r-s}, \quad 0<\zeta<1 .
\end{aligned}
$$

Therefore, we have polynomial inequalities

$$
H_{2 m, s}\left(f_{n} ; p\right) \leqq f_{n}(p)=(1-p)^{n} \leqq H_{2 m+1, s}\left(f_{n} ; p\right) \text {. }
$$

Consequently, we have corresponding Bonferroni-type inequalities, if we know explicitly the coefficients of monomials in the expression of $H_{r, s}\left(f_{n} ; p\right)$. For 
a mixed $m$, it follows from (9) that we get sharper inequalities (10) and thus sharper Bonferroni-type inequalities as $s$ increases.

\section{BONFERRONI-TYPE INEQUALITIES}

We use Newton's binomial equality to write $H_{r, s}\left(f_{n} ; p\right)$ as sum of monomials. If $r \geqq s$, then from (7) and (8),

$$
\begin{aligned}
H_{r, s}\left(f_{n} ; p\right) & =\sum_{j=0}^{s}\left(\begin{array}{l}
s \\
j
\end{array}\right)(-1)^{j} p^{j} \sum_{k=0}^{r-1}(-1)^{k}\left(\begin{array}{c}
n-s \\
k
\end{array}\right) p^{k} \\
& =\sum_{j=0}^{s}\left(\begin{array}{l}
s \\
j
\end{array}\right) \sum_{k=j}^{r-1+j}(-1)^{k}\left(\begin{array}{c}
n-s \\
k-j
\end{array}\right) p^{k} \\
& =\sum_{j=0}^{s}\left(\begin{array}{l}
s \\
j
\end{array}\right) \sum_{k=j}^{r-1}(-1)^{k}\left(\begin{array}{c}
n-s \\
k-j
\end{array}\right) p^{k}+\sum_{j=0}^{s}\left(\begin{array}{l}
s \\
j
\end{array}\right) \sum_{k=r}^{r-1+j}(-1)^{k}\left(\begin{array}{l}
n-s \\
k-j
\end{array}\right) p^{k} \\
& :=J_{1}+J_{2} .
\end{aligned}
$$

Interchanging summations, and then applying Euler's formula

$$
\sum_{i=0}^{m}\left(\begin{array}{c}
m \\
i
\end{array}\right)\left(\begin{array}{c}
n \\
k-i
\end{array}\right)=\left(\begin{array}{c}
n+m \\
k
\end{array}\right),
$$

we obtain

$$
J_{1}=\sum_{k=0}^{r-1}(-1)^{k} p^{k}\left(\begin{array}{l}
n \\
k
\end{array}\right) .
$$

Furthermore, by interchanging summations, we have

$$
\begin{aligned}
J_{2} & =\sum_{j=0}^{s}\left(\begin{array}{l}
s \\
j
\end{array}\right) \sum_{k=0}^{j-1}(-1)^{k+r}\left(\begin{array}{c}
n-s \\
k+r-j
\end{array}\right) p^{k+r} \\
& =\sum_{k=0}^{s-1}(-1)^{k+r} p^{k+r}\left[\sum_{j=1}^{s-k}\left(\begin{array}{c}
s \\
j+k
\end{array}\right)\left(\begin{array}{l}
n-s \\
r-j
\end{array}\right)\right] .
\end{aligned}
$$

Therefore, if $r \geqq s$, then

$$
H_{r, s}\left(f_{n} ; p\right)=\sum_{k=0}^{r-1}(-1)^{k}\left(\begin{array}{l}
n \\
k
\end{array}\right) p^{k}+\sum_{k=0}^{s-1}(-1)^{k+r} p^{k+r}\left[\sum_{j=1}^{s-k}\left(\begin{array}{c}
s \\
j+k
\end{array}\right)\left(\begin{array}{l}
n-s \\
r-j
\end{array}\right)\right] .
$$

Now, by (9) and (10), Theorem 1 follows from the correspondence of (1) and (2). 
Theorem 1. For positive integers $m, s$ and $n \geqq 1,2 m+s-1<n$,

$$
\begin{aligned}
& \sum_{k=0}^{2 m-1}(-1)^{k} S_{k, n}+\sum_{k=0}^{s-1}(-1)^{k}\left[\sum_{j=1}^{s-k}\left(\begin{array}{c}
s \\
j+k
\end{array}\right)\left(\begin{array}{c}
n-s \\
2 m-j
\end{array}\right) /\left(\begin{array}{c}
n \\
k+2 m
\end{array}\right)\right] S_{k+2 m, n} \\
& \leqq P\left(m_{n}=0\right) \leqq \sum_{k=0}^{2 m}(-1)^{k} S_{k, n} \\
& \quad-\sum_{k=0}^{s-1}(-1)^{k}\left[\sum_{j=1}^{s-k}\left(\begin{array}{c}
s \\
j+k
\end{array}\right)\left(\begin{array}{c}
n-s \\
2 m+1-j
\end{array}\right) /\left(\begin{array}{c}
n \\
k+2 m+1
\end{array}\right)\right] S_{k+2 m+1, n} .
\end{aligned}
$$

Furthermore, the above inequalities become sharper as $s$ increases.

For $s=0$, Theorem 1 reduces to the Bonferroni inequalities, and for $s=1$, it reduces to the Sobel-Uppuluri-Galambos inequalities (3). We list the next case $s=2$ as the following corollary.

Corollary 1. For integers $n \geqq 1,1 \leqq 2 m+1<n$,

$$
\begin{aligned}
& \sum_{k=0}^{2 m-1}(-1)^{k} S_{k, n}+\frac{2 m(2 n-2 m-1)}{n(n-1)} S_{2 m, n}-\frac{4 m(2 m+1)}{n(n-1)} S_{2 m+1, n} \\
& \quad \leqq P\left(m_{n}=0\right) \leqq \sum^{2 m}(-1)_{k=0}^{k} S_{k, n} \\
& \quad-\frac{(2 m+1)(2 n-2 m-2)}{n(n-1)} S_{2 m+1, n}+\frac{4(m+1)(2 m+1)}{n(n-1)} S_{2 m+2, n} .
\end{aligned}
$$

We now consider the case of $r \leqq s$. By (4) and (6), we have

$$
\begin{aligned}
H_{r, s}\left(f_{n} ; p\right)= & \sum_{k=0}^{r-1}\left(\begin{array}{c}
n-s \\
k
\end{array}\right) \sum_{j=k}^{s+k}\left(\begin{array}{c}
s \\
j-k
\end{array}\right)(-1)^{j} p^{j} \\
= & \sum_{k=0}^{r-1}\left(\begin{array}{c}
n-s \\
k
\end{array}\right) \sum_{j=k}^{r-1}\left(\begin{array}{c}
s \\
j-k
\end{array}\right)(-1)^{j} p^{j} \\
& +\sum_{k=0}^{r-1}\left(\begin{array}{c}
n-s \\
k
\end{array}\right) \sum_{j=r}^{s+k}\left(\begin{array}{c}
s \\
j-k
\end{array}\right)(-1)^{j} p^{j} \\
:= & J_{1}+J_{2},
\end{aligned}
$$

where interchanging summations and applying Euler's formula, we obtain

$$
J_{1}=\sum_{j=0}^{r-1}(-1)^{j}\left(\begin{array}{l}
n \\
j
\end{array}\right) p^{j}
$$

and

$$
J_{2}=\sum_{j=r}^{s-1}(-1)^{j} p^{j} \sum_{k=0}^{r-1}\left(\begin{array}{c}
n-s \\
k
\end{array}\right)\left(\begin{array}{c}
s \\
j-k
\end{array}\right)+\sum_{k=0}^{r-1}\left(\begin{array}{c}
n-s \\
k
\end{array}\right) \sum_{j=s}^{s+k}(-1)^{j}\left(\begin{array}{c}
s \\
j-k
\end{array}\right) p^{j},
$$


where the second term in the right-hand side

$$
\begin{aligned}
& =\sum_{j=s}^{r+s-1}(-1)^{j} p^{j} \sum_{k=j-s}^{r-1}\left(\begin{array}{c}
n-s \\
k
\end{array}\right)\left(\begin{array}{c}
s \\
j-k
\end{array}\right) \\
& =\sum_{j=s}^{r+s-1}(-1)^{j} p^{j} \sum_{k=0}^{r-1}\left(\begin{array}{c}
n-s \\
k
\end{array}\right)\left(\begin{array}{c}
s \\
j-k
\end{array}\right),
\end{aligned}
$$

since if $k<j-s$, then $\left(\begin{array}{c}s \\ j-k\end{array}\right)=\left(\begin{array}{c}s \\ s-j+k\end{array}\right)=0$. So

$$
J_{2}=\sum_{j=r}^{s+r-1}(-1)^{j} p^{j}\left[\sum_{k=0}^{r-1}\left(\begin{array}{c}
n-s \\
k
\end{array}\right)\left(\begin{array}{c}
s \\
j-k
\end{array}\right)\right] .
$$

Therefore, if $r \leqq s$, then

$$
H_{r, s}\left(f_{n} ; p\right)=\sum_{j=0}^{r-1}(-1)^{j}\left(\begin{array}{l}
n \\
j
\end{array}\right) p^{j}+\sum_{j=r}^{s+r-1}(-1)^{j} p^{j}\left[\sum_{k=0}^{r-1}\left(\begin{array}{c}
n-s \\
k
\end{array}\right)\left(\begin{array}{c}
s \\
j-k
\end{array}\right)\right] .
$$

By (9) and (10), and the correspondence between (1) and (2), we then have

Theorem 2. For integers $m, s$ and $n, 0 \leqq 2 m<s, 2 m+s-1<n$,

$$
\begin{aligned}
\sum_{j=0}^{2 m+1} & (-1)^{j} S_{j, n}+\sum_{j=2 m+2}^{s+2 m+1}(-1)^{j}\left[\sum_{k=0}^{2 m+1}\left(\begin{array}{c}
s \\
j-k
\end{array}\right)\left(\begin{array}{c}
n-s \\
k
\end{array}\right) /\left(\begin{array}{c}
n \\
j
\end{array}\right)\right] S_{j, n} \\
& \leqq P\left(m_{n}=0\right) \\
& \leqq \sum_{j=0}^{2 m}(-1)^{j} S_{j, n}+\sum_{j=2 m+1}^{s+2 m}(-1)^{j}\left[\sum_{j=0}^{2 m}\left(\begin{array}{c}
s \\
j-k
\end{array}\right)\left(\begin{array}{c}
n-s \\
k
\end{array}\right) /\left(\begin{array}{c}
n \\
j
\end{array}\right)\right] S_{j, n} .
\end{aligned}
$$

We list the case corresponding to $m=0$ as the following corollary.

Corollary 2. For $s \geqq 2$,

$$
\begin{aligned}
& \sum_{j=0}^{s}(-1)^{j} \frac{\left(\begin{array}{l}
s \\
j
\end{array}\right)+(n-s)\left(\begin{array}{c}
s \\
j-1
\end{array}\right)}{\left(\begin{array}{l}
n \\
j
\end{array}\right)} S_{j, n}+(-1)^{s+1} \frac{n-s}{\left(\begin{array}{c}
n \\
s+1
\end{array}\right)} S_{j, n} \\
& \leqq P\left(m_{n}=0\right) \leqq \sum_{j=0}^{s}(-1)^{j} \frac{\left(\begin{array}{l}
s \\
j
\end{array}\right)}{\left(\frac{n}{j}\right)} S_{j, n} .
\end{aligned}
$$

For $s=2$, Corollary 2 reduces to

$$
\begin{aligned}
1-S_{1, n}+2 \frac{2 n-3}{n(n-1)} S_{2, n}-\frac{6}{n(n-1)} S_{3, n} & \leqq P\left(m_{n}=0\right) \\
& \leqq 1-\frac{2}{n} S_{1, n}+\frac{2}{n(n-1)} S_{2, n},
\end{aligned}
$$

where the right-hand side is well known [2, Theorem 1.4.3, $k=n-1$ ]. 


\section{REFERENCES}

1. P. J. Davis, Interpolation and approximations, Blaisdell, New York, 1963.

2. J. Galambos, The asymptotic theory of extreme order statistics, 2nd edition, Robert Krieger, Publ. Co., Florida, 1987.

3. J. Galambos and R. Mucci, Inequalities for linear combinations of binomial moments, Publ. Math. Debrecen 27 (1980), 263-268.

Department of Mathematics, The University of Texas at Austin, Austin, Texas, 78712 